\title{
Socially Aware Integrated Centralized Infrastructure and Opportunistic Networking: A Powerful Content Dissemination Catalyst
}

Jie Hu, Lie-Liang Yang, Kun Yang, and Lajos Hanzo

The authors demonstrate that the integrated network-based content dissemination scheme outperforms its Cl-based counterpart in terms of both content delivery ratio and its various energy and delay metrics. Furthermore, the opportunistic network is capable of offloading a large fraction of tele-traffic from the overloaded Cl-based network.
The financial support of the University of Electronic Science and Technology of China, No. ZYGX2016KYQD103, is gratefully acknowledged, as is he financial support of the RC-UK's India-UK Advanced Technology Centre (IU-ATC)

${ }^{1}$ The $\mathrm{CoCl}$ may be cooperatively transmitted from multiple $\mathrm{CoCl}$ owners to unserved MUs via independent wireless channels, which results in a beneficial diversity gain due to the independent fading of the links, hence overcoming the adverse effects of the fading.

\section{ABSTRACT}

The classic centralized infrastructure (CI) exhibits low efficiency in disseminating the content of common interest across its requesters. In order to overcome the limitations of CI-based content dissemination, smart mobile devices are capable of activating direct opportunistic communications among mobile users, which returns in integrated cellular and opportunistic networks. During the content dissemination process, the social characteristics of multiple users, including their common interest in the content, their mobility patterns, their social ties, and their altruistic forwarding behaviors, should be carefully considered in order to design an efficient content dissemination scheme. We demonstrate that the integrated network-based content dissemination scheme outperforms its CI-based counterpart in terms of both content delivery ratio and its various energy and delay metrics. Furthermore, the opportunistic network is capable of offloading a large fraction of tele-traffic from the overloaded CI-based network.

\section{INTRODUCTION}

In typical scenarios multiple mobile users (MUs) are capable of forming a community of interest [1] for jointly requesting the content of common interest (CoCI). For instance, the crowd participating in the inauguration of the new Pope may form a community of interest in order to share close-up video clips of the Pope on the podium. Similarly, supporters in a football stadium may also form a community of interest so as to share video clips of a spectacular goal from different angles or the score updates from another stadium. At the time of writing, numerous mobile applications are capable of monitoring MUs' interests for the sake of disseminating their requested content and for enabling their interactions. However both the content dissemination and interactions among MUs have to be completed by the communication centralized infrastructure (CI).

CI-based content dissemination has the following three major limitations:

- Due to the sparse deployment of the CI in rural/disaster areas, MUs often suffer from intermittent connections to the CI. The CoCI can only be delivered to MUs when they enter the transmission range of the CI.

- Numerous dedicated channels have to be established in order to deliver the CoCI to multiple requesters. However, disseminating the same copies of the CoCI via dedicated channels results in a waste of precious resources and potential network congestion.

- Since the performance of the multicast/ broadcast technique is dominated by that of the "worst link" [2], as the requesters of the CoCI become denser, the "worst link" becomes even worse. As a result, CI-based multicast/broadcast often suffers from a long dissemination delay in densely populated scenarios.

During the CI-based content dissemination process, some MUs might benefit from more opportunities to contact the CI or from having better connections to the $\mathrm{CI}$ and hence succeeding in successfully receiving the CoCI earlier than their less privileged peers. As a result, activating direct communications among the MUs is capable of providing better opportunities for the unserved MUs to contact the CoCI owners and may also provide diversity gains ${ }^{1}$ in order to counteract the "worst link" phenomenon. Hence, based on the common interest of $M U s$, the opportunistic communication among the MUs themselves - either opportunistic contact or opportunistic multicast depending on the application scenarios - can be integrated into conventional CI-based content dissemination. To elaborate a little further:

- Common interests motivate MUs to share the CoCI with their hitherto unserved peers. MUs sharing common interest in a certain type of content might decide to form a community.

- Opportunistic contacts can be relied on for delivering the $\mathrm{CoCI}$ in large and sparsely populated areas. In this scenario, an unserved MU may only fetch the desired CoCI from a CoCI owner when he/she moves into the CoCI's transmission range. Opportunistic contacts can be activated without any central control by the dedicated short-range communication standards, such as 

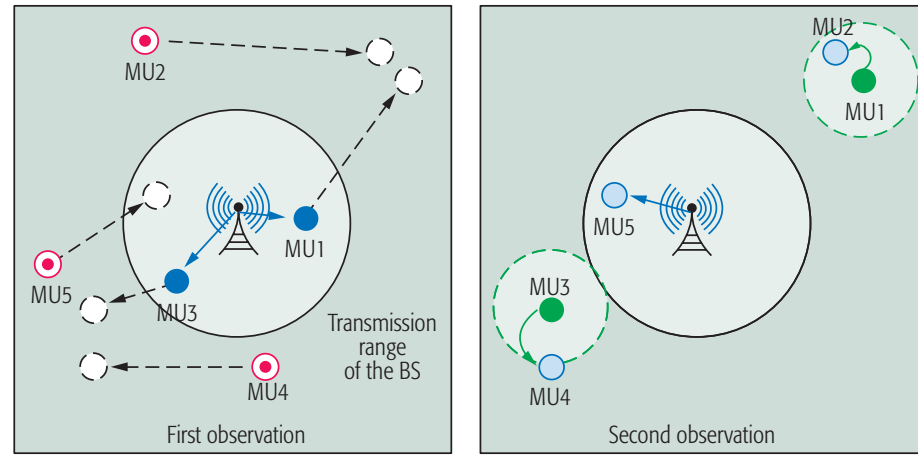

(a)

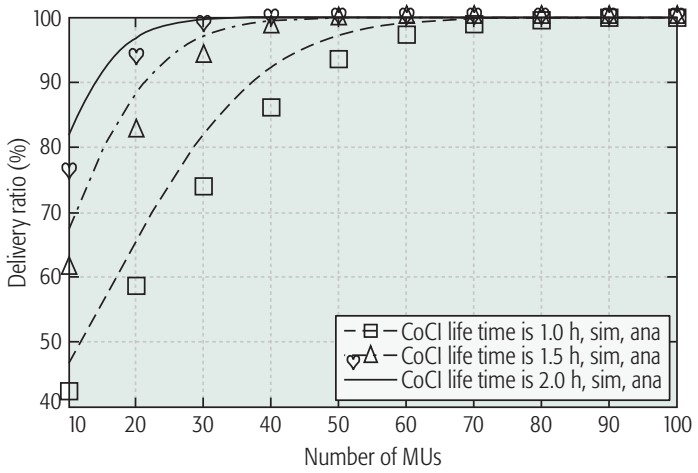

(b)

Figure 1. a) An example of the integrated network based content dissemination; b) the average delivery ratio of the integrated network-based content dissemination vs. the number of MUs, which is parameterized by the lifetime of the CoCI.

the cooperative standards conceived for interoperability, including the IEEE 802.11p amendment designed for WiFi-based direct communication, the IEEE 1609.2/3/4 standards ratified for security, network services, and multi-channel operation, and so on.

- In contrast, opportunistic multicast can be invoked for disseminating the CoCI in small and densely populated areas. In this scenario, a CoCI owner may cooperate with other peers in order to further multicast the CoCI to other hitherto unserved MUs In order to avoid interference and collisions, the opportunistic multicast should be carefully controlled by the CI, similar to the well-known LTE-assisted Wi-Fi direct technique, where the control signaling exchange is carried out by the Long Term Evolution (LTE)-based base station (BS), while the information transmission is realized by WiFi-based direct communication between a transmitter and receiver pair.

In the rest of this treatise, we detail how the above-mentioned integrated networks resolve the limitations of CI-based content dissemination. We show that the integrated network is capable of improving the intermittent connectivity in rural areas, while it is also capable of offloading tele-traffic from an overloaded CI-based network by exploiting the delay-tolerant nature of a CoCI, as discussed later. Furthermore, we elaborate on the application of the integrated network in disseminating the delay-sensitive CoCI in small and densely populated areas. A range of other applications and research challenges of our integrated network-based content dissemination regime are discussed. Finally, we conclude this article.

\section{IMPROVING THE INTERMITTENT CONNECTIVITY OF RURAL AREAS}

Due to the sparse deployment of CIs in rural/ disaster areas, MUs are not always covered adequately by the CIs, which results in intermittent connectivity.

Apart from CI-based transmission, the storecarry-and-forward protocol of [3] is adopted to increase the chances of an MU acquiring the desired CoCI, where the owner saves the CoCI in its own storage, moves within the area studied by carrying the CoCI, and then forwards it to the unserved MU within its transmission range. Both the WiFi-Direct and Bluetooth techniques support the function of discovering peer devices. Following the device discovery stage, either the CoCI owner "pushes" the CoCI to the unserved MU, or the unserved MU "pulls" the CoCI from its owner. As a result, the unserved MU does not have to continuously request the CoCI. Since the mobility-dependent contacts among MUs are not predictable, they are referred to as opportunistic contacts. Figure 1a exemplifies the content dissemination process in integrated CI and opportunistic networks. After two observations of the mobile scene, as illustrated by Fig. 1a, all five MUs have successfully received the CoCI. Among these five MUs, MU1, MU3, and MU5 receive the CoCI from the $\mathrm{CI}$ deployed in this area. If the $\mathrm{CI}$ is the sole transmitter disseminating the CoCI, only these three MUs would successfully receive the CoCI, while the other two would fail to receive it. Hence, it is plausible that the integrated CI and opportunistic network becomes capable of outperforming its CI-based counterpart in terms of its successful CoCI delivery ratio.

It is also readily recognized that opportunistic contact aided content dissemination is affected by the following factors:

Transmission range of MUs: The transmission range of MUs is jointly determined by numerous factors, including the transmit power, the statistical channel attenuation, and the capability of the error correction decoder, just to name a few. The range is often assumed to be a constant specified by the different short-range transmission techniques.

Transmission rate of opportunistic links: Once a pair of MUs enter each other's transmission range, an opportunistic link can be established for delivering information. Correspondingly, the transmission rate of this opportunistic link is determined by the specific set of parameters and techniques invoked in the physical layer as well as by the channel quality. However, if accurate power control is used, this parameter may be assumed to be near-constant.

Maximum number of opportunistic links: 
Inter-contact duration

specifies the length of a period between the instants, when a MU pair leaving each other's transmission range and when they re-enter the transmission range. $\mathrm{A}$ shorter inter-contact duration indicates that the MU pair is capable of making opportunistic contacts more frequently.

2 The delay of information transmission from the $\mathrm{Cl}$ to an MU mainly depends on the transmission rate of the Cl-aided communication link and the size of the requested files. This transmission delay is far shorter than the inter-contact duration of the MUs, which is hence reasonably assumed to be nearly instant.
Multiple opportunistic links can be established for delivering the $\mathrm{CoCI}$ from the $\mathrm{CoCI}$ owner to the unserved MUs within its coverage range. The maximum number of opportunistic links supported by the CoCI owner's device may affect the pace of the content dissemination process. However, in a large sparsely distributed area, it rarely happens that multiple unserved MUs enter the transmission range of the CoCI owner. Hence, the number of opportunistic links has a limited impact on the content dissemination in this specific scenario.

File size of the CoCI: The file size and transmission rate jointly determine the downloading time of the CoCI.

Lifetime of the CoCI: MUs are only interested in up-to-date content. Hence, after the informative lifetime of the specific content has expired, the MUs lose interest in it.

Inter-contact duration of a pair of MUs: Inter-contact duration specifies the length of a period between the instants when an MU pair leaving each other's transmission range and when they re-enter the transmission range. A shorter inter-contact duration indicates that the MU pair is capable of making opportunistic contacts more frequently.

Contact duration of a pair of MUs: Contact duration specifies the period in which an MU pair is within each other's transmission range. A longer contact duration may increase the chance of an MU pair completing a file transfer between each other.

By jointly considering all the aforementioned factors, an effective contact between an unserved MU and a CoCI owner is defined by simultaneously satisfying the following two conditions:

- The unserved MU enters the transmission range of the CoCI owner.

- The unserved MU stays within the transmission range of the CoCI owner for a period longer than the sufficient downloading time of the $\mathrm{CoCI}$.

The content dissemination process in the integrated network can be modeled by a continuous-time pure-birth Markov chain (CT-PBMC) [3]. Relying on the CT-PBMC, we are capable of analyzng the various delay metrics as well as average successful delivery ratio, also bearing in mind when the CoCI expires.

Let us now present a crisp performance characterization. Observe from Fig. $1 b$ that if more MUs participate in the integrated network-based content dissemination, the average delivery ratio is substantially improved. Furthermore, if the informative lifetime of the CoCI is prolonged, more MUs may successfully receive the desired CoCI before it expires.

\section{OfFlOADING Tele-TRAFFiC FROM THE Cl}

In contrast to the rural/disaster scenario studied in the previous section, we assume adequate coverage provided by the CI deployed in the large area studied, where the MUs are always capable of maintaining reliable connections to the CI.

In this scenario, we can reasonably assume that the CoCI can be nearly instantly disseminated by the CI across the target community of interest. ${ }^{2}$ This CI-based approach requires having dedicated channels established between the MUs and their associated CI. However, disseminating the same copies of the CoCI via the limited number of dedicated channels imposes an increased burden on the CI. Fortunately, as discussed earlier, a CoCI may attract the interest of the MUs for the duration of its lifetime. MUs do not have to receive the CoCI at the same time. In contrast, disseminating the CoCI before it expires is expected to satisfy the requesters. As a result, exploiting the delay-tolerant nature of the CoCI, the opportunistic contact-based communication among the MUs can be relied on for delivering the CoCI to a large fraction of its requesters, which hence offloads tele-traffic from the overloaded CI. Furthermore, offloading tele-traffic from a high-power CI to a low-power opportunistic contact-based transmission is capable of significantly reducing energy consumption.

As detailed in [4], the optimal content dissemination approach may be described by the following procedure:

- Step 1: The CI initially transmits the CoCI to some of the MUs in the opportunistic network.

- Step 2: The CoCI is spontaneously disseminated via opportunistic contact-based communications among the MUs before it expires.

- Step 3: At the very end of the CoCl's lifetime, the $\mathrm{CI}$ is invoked again for delivering the CoCI to the hitherto unserved MUs.

In order to offload as much tele-traffic from the CI to the opportunistic networks as possible in step 2, our main task is to find an optimal MU receiver with members initially receiving the CoCI from the CI in step 1.

Social network analysis assists us in identifying the most significant MUs in an opportunistic network. However, we have to first model the social ties among the MUs according to their contact patterns. As shown in Fig. 2a, the shaded boxes represent the contact duration between a pair of MUs. Several conventional metrics, that is, the contact frequency, average inter-contact duration, and average contact duration, have been recorded for characterizing how socially close a pair of MUs may be deemed to be according to their contact pattern. However, these conventional metrics cannot always accurately specify the social closeness of a pair of MUs. For example, observe from Fig. 2a that the pair of MUs associated with the contact pattern of (a-2) has a weaker social connection than those associated with (a-3) and (a-4) in terms of the contact frequency, average inter-contact duration, and average contact duration. However, these metrics all fail to characterize the difference between cases (a-3) and (a-4). In order to jointly consider the regularity of contact patterns with the above-mentioned conventional metrics, the so-called social pressure metric (SPM) was proposed in [5] for accurately modeling the social strength of a pair of MUs. Explicitly, given a specific period $T$, the SPM between $M U_{i}$ and $M U_{j}$ is defined as $S P M_{i j}=\left(\sum_{x=1}^{n} t_{\text {inter }, x}^{2}\right) /(2 T)$, where $t_{\text {inter } x}$ is the $x$ th inter-contact duration. The reciprocal of the SPM represents the weight of the social tie. With the aid of this SPM, we can finally decide that the pair of MUs associated with (a-4) has stronger social strength than that asso- 


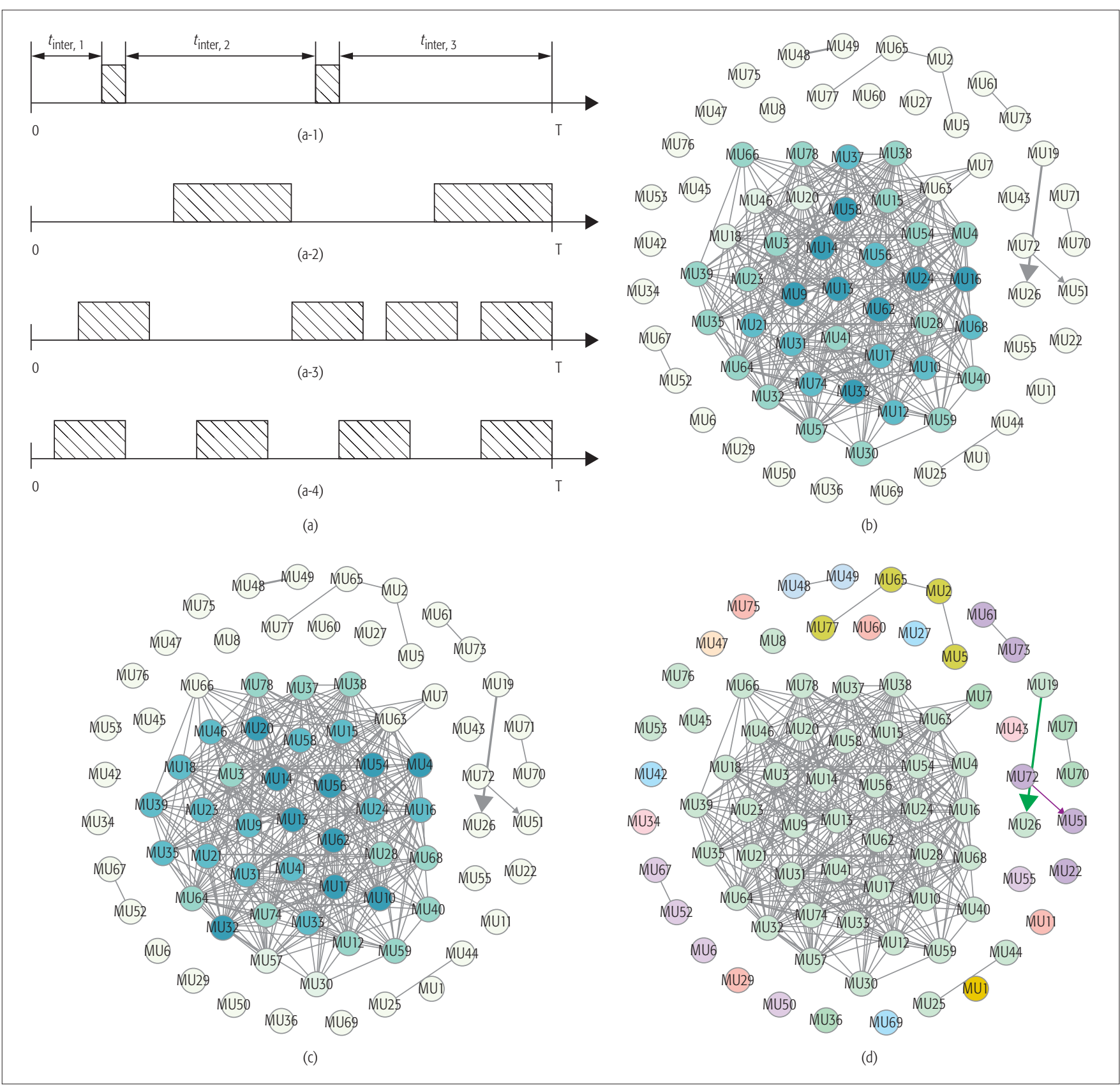

Figure 2. a) Different contact examples among MUs; b-d) the social network extracted from the mobility traces during 8:00-9:00 a.m. on the opening day of INFOCOM 2006, where the arrows represent the direction of the social ties. The nodes of the resultant social network are colored according to b) their out-degree centralities; c) their betweenness centralities; d) the components to which they belong.

ciated with (a-3).

Real-life social ties do correspond to the contact pattern of MUs. For example, people sharing strong social ties are more likely to be found in each other's proximity. The contact pattern of MUs may change their real-life social ties. For example, people frequently being in each other's proximity may indeed strengthen their social ties, while people rarely visiting each other may typically weaken their social ties. As a result, our SPM modeling closely reflects the relationship between the MUs' contact pattern and their reallife social ties.

After evaluating the weight of the social ties between all pairs of MUs, we only retain the specific social ties having a higher weight than a pre- defined threshold. The resultant social network is uniquely and unambiguously characterised by a directed and weighted graph. ${ }^{3}$ Relying on the toolbox of social network analysis, we can finally rank all the MUs according to their social significance. An example of a social network is portrayed in Figs. 2b-2d, obtained by analyzing the contact patterns of MUs during the hour spanning from 8:00 to 9:00 a.m., on the opening day of INFOCOM 2006. Then the following three schemes may be conceived for selecting the initial MU receiver set by considering the MUs' social significance:

Out-degree-based selection: The out-degree of an MU is defined as the number of social links that emerges from this MU and terminates at

\footnotetext{
3 The contact-pattern-dependent social tie of a pair of MUs is said to be directed, which is a consequence of the heterogeneous functionality of mobile devices. For example, MU A may find MU B within its transmission range, and hence MU A may deliver information to MU B. However, the reverse of this may not be true, since $\mathrm{MU}$ $B$ may have a shorter transmission range than MU A.
} 


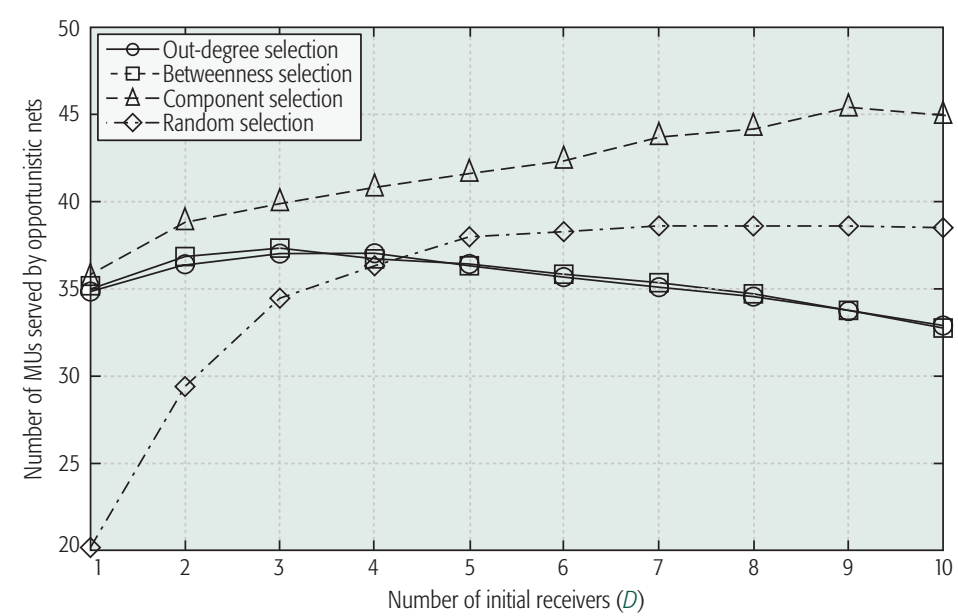

Figure 3. The size of the initial receiver set vs. the number of MUs served by the opportunistic network. Contact patterns of the 78 mobile users are extracted from the mobility traces during 8:01-9:00 a.m. on the opening day of INFOCOM 2006. The opportunistic contacts between an arbitrary pair of MUs are activated by their Bluetooth devices, and the transmission rate of the opportunistic link is $1 \mathrm{Mb} / \mathrm{s}$. Bluetooth is a half-duplex peer-topeer communication technique. Hence, while a source is transmitting data to a target, no extra links can be established, even if another unserved MU enters the transmission range of the source. The file size of the CoCI is 40 Mbits (5 MB). initial receiver set is randomly selected without specifically considering any social metrics.

Figure 3 exemplifies the capability of the opportunistic contact-based communication of the MUs offloading the tele-traffic from the CI-based communication. Please refer to [3] for the detailed simulation settings of the scenario we would like to characterize next. Observe from Fig. 3 that the component-based selection outperforms all its counterparts in terms of its ability to offload the tele-traffic. According to both the out-degree-aided and the betweenness-based selections, the MUs of the initial receiver set all belong to the largest component. Hence, they have similar performance, as shown in Fig. 3. Furthermore, when the size of the initial receiver set is small, random selection performs worst, because it is likely to select isolated MUs. However, when the size of the initial receiver set is large, random selection becomes the second best scheme, because its initial receiver set is capable of covering many isolated components.

\section{EFFICIENT CONTENT DISSEMINATION IN Densely Populated Areas}

In the scenarios shown earlier, where the MUs are sparsely distributed in a large area, the delay-tolerant nature of the CoCI and the opportunistic contact among the MUs are relied on for disseminating the CoCI across the set of requesters. In this section, a different scenario is considered, with the main characteristics summarized as follows:

- The MUs are densely distributed in a small area. The size of this small area is comparable to the transmission range of the MUs.

- An MU is connected to any of its peers by a single-hop/multihop link.

- The CoCI in this scenario is delay-sensitive.

CI-based multicast constitutes an effective technique for disseminating the delay-sensitive CoCI across its coverage area. When the population of MUs requesting the CoCI becomes dense, even CI-based multicast becomes inefficient. Due to its quality of service (QoS)-guaranteed nature, the performance of the CI-based multicast is dominated by the worst link in the set of links connecting this CI to all the requesters [2]. Since the MUs are more densely populated, the probability of the worst link becoming much worse is increased, as shown in Fig. 4a. As a result, CI-based multicast suffers from a long delay during disseminating the CoCI to all its requesters. Furthermore, during the CoCI dissemination process, the CI has to repeatedly multicast the CoCI until all the requesters successfully receive it; hence, the CI fails to satisfy other communication demands, which results in inefficient exploitation of the CI.

We can exploit the following facts in order to improve the CoCI delivery. During CI-based multicast, some of the MUs benefiting from statistically better channels may successfully receive the CoCI much earlier than their less privileged peers. If we could exploit the redundant copies of the CoCI in the storage buffer of these successful MUs and hence activate direct peer-to-peer ( $\mathrm{P} 2 \mathrm{P})$ communications between them and their hitherto unserved peers, we may 

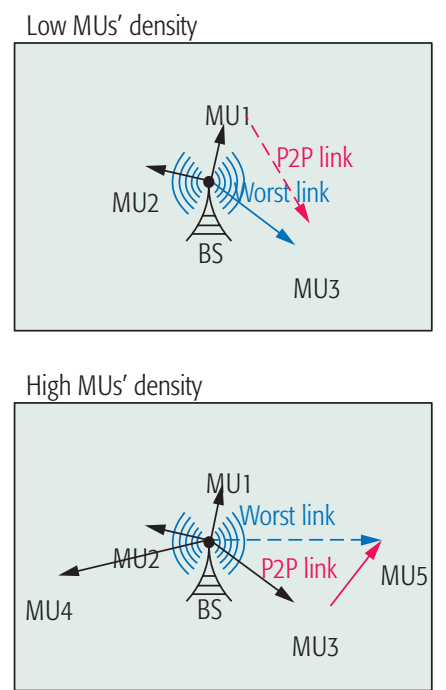

(a)

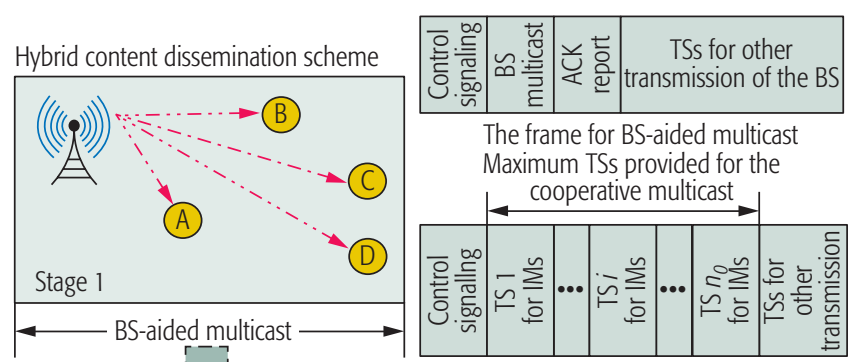

The frame for cooperative multicast

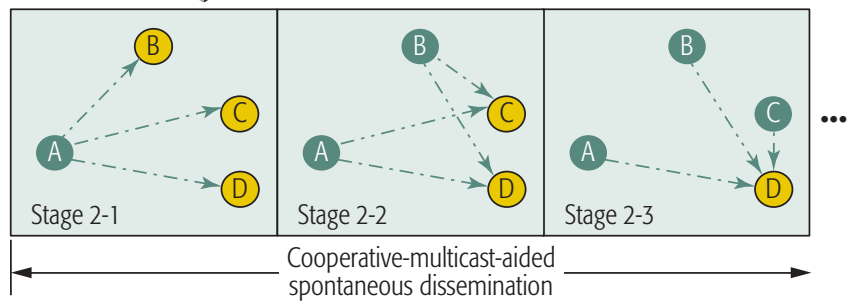

(b)

Figure 4. a) Activating P2P link among MUs overcomes the "worst link" phenomenon; b) the hybrid content dissemination scheme and the TDMA-based frame structure.

increase the diversity gain of CoCI delivery and thus overcome the "worst link" phenomenon often encountered in densely populated areas, as shown in Fig. 4a. Furthermore, we may also relieve the $\mathrm{CI}$ from the cumbersome multicasting task. Since we cannot predict which specific MUs may successfully receive the CoCI first, opportunistic multicasting is invoked for activating the P2P communications among the MUs by exploiting the broadcast nature of wireless channels. However, as also portrayed in Fig. 4a, we should note that if the number of requesters is not very high, activating $\mathrm{P} 2 \mathrm{P}$ communications may not necessarily produce a better link than the worst link of CI-based multicast, so it may not improve the speed of content delivery.

In order to cooperatively disseminate the $\mathrm{CoCI}$ in densely populated areas, MUs form a community of interest by obeying the following conditions:

- MUs share a common interest in the same content.

- The CoCI is delay-sensitive.

- MUs roam within a bounded area having a relatively small size.

Let us elaborate a little further on modeling the MUs' interests. The statistics of users' Youtube viewing behaviors suggest that a small fraction of popular contents attract the interest of a large fraction of users [6]. We model an MU's interest in a specific piece of content $\mathcal{C}_{i}$ by the probability $\operatorname{Pr}\left(\mathcal{C}_{i}\right)$ of this $\mathrm{MU}$ requesting $\mathcal{C}_{i}$ from the CI. If we have a range of contents $\mathcal{C}_{i} \mid i=1$, ... $\mathcal{M}$, their corresponding requesting probabilities $\left.\operatorname{Pr}\left(\mathcal{C}_{i}\right) \mid i=1, \ldots, \mathcal{M}\right\}$ obey the Zipf distribution [7].

As shown in Fig. 4b, we characterize our integrated-network-aided hybrid content dissemination scheme in the scenario of densely populated areas. In the first stage of our regime, CI-based multicast is invoked for initially disseminating the CoCI to all the requesters. CI-based multicasting will be curtailed once at least a single
MU requester successfully received it. Then, the CoCI owners cooperate with each other in order to disseminate the CoCI to the hitherto unserved MUs with the aid of opportunistic multicasting. Once an unserved MU successfully received the CoCI, it will join the CoCI-owner set and it will further multicast the CoCI during the next stage.

In order to avoid any unexpected collision and interference, a time-division multiple access (TDMA) scheme ${ }^{4}$ is implemented in the medium access control (MAC) layer. The related transmission frame structure is also portrayed in Fig. $4 \mathrm{~b}$, where a single time slot of a transmission frame only allows a single transmitter to forward the CoCI. Classic round-robin scheduling can be applied in the MAC layer due to its low-complexity nature. However, for the sake of further improving the attainable performance of the CoCI dissemination, we could carefully select the most suitable CoCI owners for forwarding the CoCI to the hitherto unserved MUs during the next stage of CoCI dissemination. As a result, we should evaluate the potential impact of a CoCI owner on all the unserved MUs with the aid of social network analysis tools [8]. During the second stage of the cooperative multicast aided CoCI dissemination portrayed in Fig. 4, the CI plays the role of a central controller in order to facilitate control signaling exchange and to efficiently schedule the transmissions of multiple CoCI multicasters.

In the physical layer, the channel effects are modeled by both the uncorrelated Rayleigh fading and the path loss. Since the MUs roam across the area studied, the path loss between the CI and an MU as well as that between a pair of MUs varies. The movement of MUs in this scenario can be modeled by the ubiquitous uniform mobility model, which has been widely adopted for the performance analysis of mobile ad hoc networks (MANETs) [9].

Furthermore, we shall incorporate MUs' altruistic behavior into the modeling of the
Despite the above

mentioned challenges, our integrated $\mathrm{Cl}$ and opportunistic networks may find numerous applications in practical scenarios. Apart from the content dissemination process designed for distributing the $\mathrm{CoCl}$, our integrated networks may also be applied in disaster areas for uploading important messages.

${ }^{4}$ Other orthogonal multiple access protocols, such as frequency-division multiple access (FDMA), orthogonal FDMA (OFDMA), and code-division multiple access (CDMA), can also be adopted for providing orthogonal channels. 


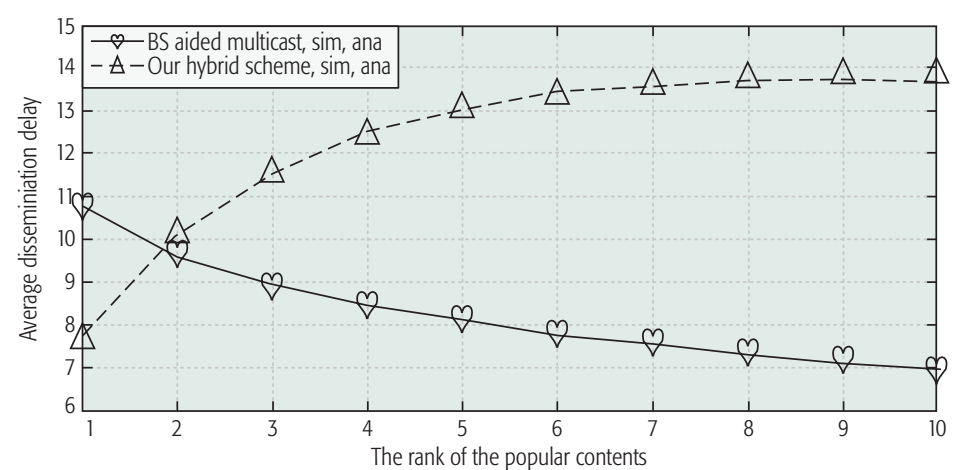

Figure 5. Average content dissemination delay vs. content popularity.

opportunistic multicast aided content dissemination process $[10,11]$. We assume that at the beginning of each sub-stage portrayed in Fig. $4 \mathrm{~b}$, the CoCI owners may independently make a decision as to whether they are willing to forward the CoCI received with a probability of $q$, which is defined as the factor of altruism (FA). The MUs' altruistic behavior is affected by numerous factors, such as the users' interest in the content, their energy concerns, and a range of other random factors.

Apart from the MUs' altruistic behavior, the social ties among the MUs may also constrain the multicasting of the CoCI. Due to security and privacy concerns, an MU is only willing to share the CoCI with its social contacts. As demonstrated by [12], people's social ties are largely determined by their geographic distances. The probability $p$ of a pair of persons sharing a social tie is proportional to $d^{-\alpha}$, where $d$ is the distance between this pair of persons, and $\alpha$ is the associated social exponent. By further considering the small world phenomenon exhibited by social networks, the social contacts of an MU are divided into short-range contacts and long-range contacts $[13,14]$. Short-range contacts of an MU are formed by all the MUs within the neighborhood range of the target MU, while long-range contacts of an MU are formed by the MUs outside the neighborhood range of the target MU with a probability proportional to $d^{-\alpha}$.

In Fig. 5, we study the impact of content popularity on the average content dissemination delay when all the requesters successfully receive the CoCI. The CI in this example is a typical BS. We set the FA to 0.5 and the total number of MUs roaming within the area studied to $\mathcal{N}=$ 100. Furthermore, 10 different pieces of content are considered in this scenario, with rank one being the most popular piece and rank 10 being the least popular piece. Observe from Fig. 5 that our hybrid content dissemination scheme outperforms BS aided multicast when disseminating the most popular content. However, BS aided multicast still constitutes a more efficient option of disseminating the less popular pieces of content.

\section{OPEN RESEARCH ISSUES AND OtHER APPLICATIONS}

Several open problems still remain to be solved in the design of our integrated network-based content dissemination regime.

In order to accurately estimate the associated social properties, MUs have to have an awareness of the global network and have to intelligently process this knowledge on their own devices. However, in a purely distributed network, this awareness can only be acquired by information exchange among MUs by relying on their opportunistic contacts, which is a slow process consuming a large amount of energy. Furthermore, the computing capability of a mobile device may turn out to be too limited to process the required knowledge right across the global network. In order to overcome these inefficiencies, MUs may rely on their specific "virtual machines" in the mobile cloud for exchanging information, sharing storage space, and carrying out computing tasks.

The MUs' altruistic behaviors largely depend on the intensity of their interest. However, the correlation between the MUs' interests and their resultant altruistic behaviors remains a mystery. Their mutual influence has to be translated into a mathematical model. Furthermore, the altruistic behavior can be viewed as a strategy adopted by an individual MU for striking a balance between the associated delay reduction and the energy dissipation. Game theory may come to serve for finding the optimal solution.

In order to compensate for the energy loss of MUs, we can enable them to harvest additional energy during the content dissemination process. For example, the device of an MU may harvest energy from the environment as a solar cell does. Alternatively, simultaneous information and energy transfer can be used during the content dissemination process, where the intermediate relay may harvest additional energy from the RF signal, as a reward for forwarding the content.

Disseminating the delay-sensitive content across large areas may impose other challenges. Due to the slow movement of the CoCI owners, their local multicasting cannot forward the CoCI to the set of distant and hitherto unserved MUs until they enter the proximity of their unserved peers. Therefore, the CoCI owners may become clustered, so none of them can efficiently forward the CoCI to the distant unserved MUs. In order to guard against this clustering effect, novel resource scheduling techniques may be designed by jointly considering both the CI's resources and the MUs' resources.

Despite the above mentioned challenges, our integrated CI and opportunistic networks may find numerous applications in practical scenarios. Apart from the content dissemination process designed for distributing the CoCI, our integrated networks may also be applied in disaster areas for uploading important messages. Furthermore, our integrated networks may be applied in vehicular ad hoc networks in order to disseminate important traffic information and facilitate the design of smart vehicles. Furthermore, our integrated networks may be introduced into aeronautical ad hoc networks in order to implement low-complexity information exchange among aircraft. Moreover, they may also be implemented in some communities, such as a university campus, railway stations, and airports, in order to disseminate important information. Last but not least, our integrated networks may improve the 
charity project One Child One Laptop in order to further reduce the expense of delivering educational programs.

\section{CONCLUSIONS}

In this treatise, we study content dissemination in integrated CI and opportunistic networks by exploiting MUs' common interest and activating their opportunistic communications. With the aid of integrated networks, we may significantly increase the average successful delivery ratio of the CoCI and offload tele-traffic from the CI to the opportunistic network. We can also substantially reduce the content dissemination delay, and may reduce the total energy dissipated by exhaustively disseminating the $\mathrm{CoCI}$ to all its requesters, since the CI-based high-power transmission is offloaded to the low-power direct transmission among MUs. However, as the price of achieving the above mentioned benefits, the MUs have to rely on their own energy in order to assist in the content dissemination process, while the control overhead imposed by scheduling the direct communications among the MUs has to be increased. Interested readers may refer to the seminal references listed in Table 1 for more technical details.

\section{REFERENCES}

[1] F. Henri and B. Pudelko, "Understanding and Analysing Activity and Learning in Virtual Communities," J. Computer Assisted Learning, vol. 19, no. 4, Dec. 2003, pp. 474-87.

[2] I.-H. Lee, H. Lee, and H.-H. Choi, "Exact Outage Probability of Relay Selection in Decode-and-Forward Based Cooperative Multicast Systems," IEEE Commun. Letters, vol. 17, no. 3, Mar. 2013, pp. 483-86.

[3] J. Hu et al., "Bridging the Social and Wireless Networking Divide: Information Dissemination in Integrated Cellular and Opportunistic Networks," IEEE Access, vol. 3, Oct. 2015, pp. 1809-81.

[4] V. Sciancalepore et al., "Offloading Cellular Traffic Through Opportunistic Communications: Analysis and Optimization," IEEE JSAC, early access, July 2015.

[5] E. Bulut and B. K. Szymanski, "Exploiting Friendship Relations for Efficient Routing in Mobile Social Networks," IEEE Trans. Parallel and Distrib. Sys., vol. 23, no. 12, Dec. 2012, pp. 2254-65.

[6] M. Zink et al., "Characteristics of YouTube Network Traffic at a Campus Network - Measurements, Models, and Implications," Computer Networks, vol. 53, no. 4, Mar. 2009, pp. 501-14.

[7] M. Taghizadeh et al., "Distributed Cooperative Caching in Social Wireless Networks," IEEE Trans. Mobile Computing, vol. 12, no. 6, June 2013, p. 1037-53.

[8] J. Hu, L.-L. Yang, and L. Hanzo, “Cooperative Multicast Aided Picocellular Hybrid Information Dissemination in Mobile Social Networks: Delay/ Energy Evaluation and Relay Selection," 2014 IEEE Wireless Commun. and Networking Conf., Istanbul, Turkey, Apr. 2014, pp. 3207-12.

[9] H. Zhang, Z. Zhang, and H. Dai, "Gossip-based Information Spreading in Mobile Networks," IEEE Trans. Wireless Commun., vol. 12, no. 11, Nov. 2013, pp. 5918-28.

[10] J. Hu, L.-L. Yang, and L. Hanzo, "Mobile Social Networking Aided Content Dissemination in Heterogeneous Networks," China Commun., vol. 10, no. 6, June 2013, pp. 1-12.

[11] --, "Delay Analysis of Social Group Multicast Aided Content Dissemination in Cellular System," IEEE Trans. Commun. ???

[12] D. Liben-Nowell et al., "Geographic Routing in Social Networks," Proc. Nat'l. Acad. Sci. U.S.A., vol. 102, no. 33, Aug. 2005, pp. 11623-28.

[13] J. Hu, L.-L. Yang, and L. Hanzo, "Throughput and Delay Analysis of Wireless Multicast in Distributed Mobile Social Networks Based on Geographic Social Relationships," 2014 IEEE Wireless Commun. and Net. Conf., Apr. 2014, pp. 1874-79.

[14] --, "Distributed Cooperative Social Multicast Aided Content Dissemination in Random Mobile Networks," IEEE Trans. Vehic. Tech., vol. 64, no. 7, July 2014, pp. 3075-89.

\section{BIOGRAPHIES}

JIE Hu (hujie@uestc.edu.cn) bios needed

LIE-LIANG YanG (lly@ecs.soton.ac.uk) bios needed

Kun YANG (kunyang@essex.ac.uk) bios needed

\begin{tabular}{|c|c|c|}
\hline Year & Author & Contribution \\
\hline 2005 & $\begin{array}{l}\text { Liben-Nowell et } \\
\text { al. [12] }\end{array}$ & $\begin{array}{l}\text { Revealed that social ties among online social networking users } \\
\text { depend on their geographic distances. }\end{array}$ \\
\hline 2012 & Bulut et al. [5] & $\begin{array}{l}\text { Proposed social pressure metric for characterizing the social strength } \\
\text { among MUs according to their contact patterns. }\end{array}$ \\
\hline 2013 & Hu et al. [10] & $\begin{array}{l}\text { Provided a detailed survey on mobile social networking aided con- } \\
\text { tent dissemination in heterogeneous networks }\end{array}$ \\
\hline \multirow{3}{*}{2014} & Hu et al. [8] & $\begin{array}{l}\text { Proposed a social network analysis aided resource scheduling } \\
\text { scheme for the cooperative multicast aided content dissemination. }\end{array}$ \\
\hline & Hu et al. [13] & $\begin{array}{l}\text { Analyzed the performance of the single-hop non-cooperative multi- } \\
\text { cast protocol by considering the geographic social ties among MUs. }\end{array}$ \\
\hline & Hu et al. [14] & $\begin{array}{l}\text { Studied the multi-stage cooperative social multicast aided content } \\
\text { dissemination by considering the geographic social ties among MUs. }\end{array}$ \\
\hline \multirow{2}{*}{2015} & Hu et al. [3] & $\begin{array}{l}\text { Studied the content dissemination and its applications in integrated } \\
\text { cellular and opportunistic networks for the large-scale and small-scale } \\
\text { scenarios. }\end{array}$ \\
\hline & Hu et al. [11] & $\begin{array}{l}\text { Studied the delay performance of the social group aided content } \\
\text { dissemination in cellular systems by considering the MUs' altruistic } \\
\text { behaviors. }\end{array}$ \\
\hline
\end{tabular}

Table 1. Seminal references for further reading.

LAJOS HANZO (lh@ecs.soton.ac.uk) bios needed 
